\title{
Meals in the melting-pot: Immigration and dietary change in diversifying cities
}

\author{
Nicola Frances Rule ${ }^{a, *}$, Colin Charles Dring ${ }^{b}$, Thomas F. Thornton ${ }^{c}$ \\ ${ }^{a}$ University of Oxford, School of Geography and Environment, Environmental Change Institute, UK \\ ${ }^{\mathrm{b}}$ University of British Columbia, Faculty of Land and Food Systems, Centre for Sustainable Food Systems, Canada \\ ${ }^{c}$ University of Alaska Southeast, Alaska Coastal Rainforest Center, Juneau, AK, USA
}

\section{A R T I C L E I N F O}

\section{Keywords:}

Dietary acculturation

Dietary change

Immigration

Food practices

Food environment

Urban diets

\begin{abstract}
A B S T R A C T
Changes in diets and food practices have implications for personal and planetary health. As these implications have become more apparent, dietary change interventions that seek to promote healthy and sustainable transitions have proliferated, and the processes and drivers of dietary change have come under increasing scrutiny. In particular, dietary acculturation has been recognised as a driver of dietary change in the context of immigration to expanding, cosmopolitan cities. However, research has largely focused on changes in the diets of immigrants and ethnic minorities. In contrast, this study contributes to our understanding of the process of dietary acculturation among the largest population groups in Vancouver, Canada - Chinese- and European-Canadians - in the context of the rapid diversification of the population and food environments in this city. This is done through the analysis of descriptive and contextualised interview and observational data, and a focus on social practices. These data show that food practices, particularly in cosmopolitan urban contexts, are constantly in flux, as diverse ethnic groups come into contact, and new generations develop their own hybrid food cultures. By demonstrating and theorising this process of dietary acculturation, this research offers insights how cultural interactions relate to dietary transitions. It presents an exploratory model for considering how food practices change through dietary acculturation, which is relevant to the design of interventions that aim to support healthier and more sustainable dietary transitions.
\end{abstract}

\section{Introduction}

The last two decades have marked a growing awareness that dietary choices have important consequences for both personal and planetary health (Conijn et al., 2018; Poore \& Nemecek, 2018; Popkin, 2006; Steffen et al., 2015). As a result, there has been increasing interest in understanding how and why people make decisions about their diets, the processes of dietary change, and the sociocultural factors that influence these changes. This research offers insight into these processes of dietary change through the lens of acculturation and offers a methodological approach for asking questions about how cultural interactions play out in dietary transitions.

One important factor affecting the diets of individuals and communities is their food environment - the spatial distribution, accessibility, and affordability of different types of foods and food sources (National Collaboration Centre for Environmental Health (NCCEH), 2017). Food environments arise out of the interactions between the biophysical environment, political and economic systems, and sociocultural practices (Zimmerer, 2018), and include the places where food is procured, processed, and consumed (Chen \& Kwan, 2015). While food environment research often focuses on food retail, other components of individuals' food environments include restaurants, food gardens, household and community kitchens, and shared meals.

As human societies have become more globalised and more urbanised, food environments have shifted away from being largely shaped by locally produced foods (Crowther, 2013). For example, as migration to

\footnotetext{
* Corresponding author. Present address: ICLEI Africa, 14 Bridge Boulevard, Century City, Cape Town, 7441, South Africa.

E-mail address: Nicola.Rule@alumni.uct.ac.za (N.F. Rule).
} 
the Global North from Africa, Asia and Latin America has increased in the last three decades (Migration Data Portal, 2021; Organisation for Economic Cooperation and Development (OECD), 2019), many cities have become home to large and well-established ethnic minority populations. ${ }^{1}$ These populations (like the Bengali population in London, United Kingdom, or the Chinese population in Vancouver, Canada), have a substantial impact on the local food environment. The Canadian census does not track food service establishments by race or ethnicity. In Vancouver, for example, food environment changes associated with the ongoing diasporic immigration and establishment of these populations have included the opening of shops and restaurants dedicated to ethnic foods $s^{2}$ in parts of the city where these foods were not previously available, and the increasing availability of ethnic foods in 'mainstream' shops and restaurants (Cheung, 2018; Rule, 2018).

The diversification of urban populations through immigration has led not only to interest in how food environments change, but also how food practices and diets change. Dietary acculturation is the process through which people's dietary choices and food practices change as they come into contact with a new cultural environment. This includes the adoption of new foods or materials into food practices, as well as changes to the competences and meanings associated with these food practices. As with much of the historical literature on other forms of acculturation and cultural contact (see, for example, Hallowell, 1959) the dietary acculturation literature has been preoccupied with how perceived 'dominant' Euro-American cultures have influenced the food practices of perceived 'sub-dominant' ethnic minorities. This is particularly evident from studies on how the dietary patterns of immigrants from the Global South change when they move to European and North American cities. The most frequently described trend is of immigrants increasing their consumption of processed foods and refined carbohydrates - increasing the risk of diet-related preventable diseases - when they move (Ayala et al., 2008; Sanou et al., 2014). As Hallowell (1959) and others point out (Huang, 2019; Ye, 2018), however, acculturation is a reciprocal or bi-directional process, or rarely ever fully achieved (Rouse, 1995). In addition, the process of acculturation varies considerably between groups and depending on contexts. Multiple studies examine the gradual loss of cultural identity (Satia-Abouta et al., 2002; Vallianatos \& Raine, 2008) while others note that individuals can have multiple identities (Chapman \& Beagan, 2013; Das Gupta, 1997; Rouse, 1995), preserving or modifying certain practices to resist both acculturation in the host country and Westernization in country of origin.

Using descriptive, contextualised data, this study explores dietary acculturation from an alternative angle. We ask whether and how the diets and food practices of a city's largest population groups change as immigration causes the food environments and population of that city to diversify. Using Vancouver, Canada as a study site, we investigated how the experiences and food practices of both European-Canadian and Chinese-Canadian residents, across generations, have been influenced and transformed through acculturation. An analytical framework derived from social practice theory was used and is described in Section 1.1. Details of the site and reasons for its selection are given in Section 1.2 .

\footnotetext{
1 The authors acknowledge that it is problematic to use the term 'ethnic' to describe only what is associated with ethnic minority groups while not ascribing ethnicity to white Europeans. As described in Section 2 the participants in the study are grouped by race (Asian-Canadian and White-Canadian) rather than ethnicity. In this paper, the term 'ethnic minority' is used to refer generally to non-European and non-indigenous population groups, called 'visible minorities' by Statistics Canada.

2 The term 'ethnic foods' is used to refer to ingredients and dishes typically associated with an ethnic minority population, following the common usage of the term.
}

\subsection{Analytical framework}

Social practice theory provides a particularly useful framework for studying the relationship between individual dietary choices and collective trends of dietary change, because it uses the change and perseverance of patterns of action - social practices - as the starting point for analysis (for example in Twine, 2018). This addresses the limitations of an approach that focuses on individuals and rational decision-making (de Boer \& Aiking, 2017; Greene et al., 1999; Rothman et al., 2009) by accounting for the roles of context and shared social schemata in driving dietary change.

A social practice can be understood as "a temporally unfolding and spatially dispersed nexus of doings and sayings" (Schatzki, 1996, p. 89). These "doings and sayings" are performed by people in different contexts, but are united into a particular social practice by common elements (Table 1). The scheme of elements of practice developed by Shove et al. (2012) is used in this study to understand and analyse changes in food practices. In this scheme, social practices are comprised of materials, meanings, and competences, and changes in practices are caused by changes in these elements.

In order to investigate broadly how food practices have been impacted by changes in the food environments and ethnic diversity of Vancouver, domains of practice rather than individual social practices are analysed in this study. A domain of practice is a context or area of people's lives in which any of a range of social practices can be performed (Rule, 2018). Practices within a domain are united by similar meanings, competences, and materials, but the actions they involve are too diverse to be considered a single practice. For example, shopping for food is a domain of practice in which some people may order groceries online, others may drive to a large supermarket once a month, and still others may walk to the local farmers' market. These practices may involve similar materials (e.g. money and vegetables), meanings (e.g. being associated with provision), and competences (linked to choosing what to eat). However, the practices also differ significantly, in terms of the knowledge-practice-belief complexes to which they belong (Berkes, 2012; e.g. links to driving, surfing the internet, or socialising with neighbours) and in terms of the food environments they are associated with.

\subsection{Study site}

Vancouver is the most populous city in what is dominantly called British Columbia, Canada located in the traditional, ancestral, and unceded territories of the Skwwwú7mesh (Squamish), Səlílwəta?/Selilwitulh (Tsleil-Waututh) and $\mathrm{x}^{\mathrm{w} m}{ }^{\mathrm{m}} \theta \mathrm{k}^{\mathrm{w}}$ əyəm (Musqueam) Nations. British immigrants started to settle in the area from the mid-nineteenth century, with many Chinese labourers coming to British Columbia to work on railway construction (Holland, 2007). In 1886, the City of Vancouver was incorporated into the Colony of British Columbia, by which time people of both European origin (European-Canadians) and Chinese origin (Chinese-Canadians) were farming in the area (Gibb \& Wittman, 2013). As a result, the global influences on Vancouver's food system date back nearly a century and a half.

Table 1

The three elements of social practices described by Shove et al. (2012).

\begin{tabular}{|c|c|c|}
\hline Element & Description & Examples \\
\hline Materials & $\begin{array}{l}\text { The physical things necessary } \\
\text { to perform a practice }\end{array}$ & $\begin{array}{l}\text { Ingredients; recipes; kitchen } \\
\text { equipment }\end{array}$ \\
\hline Meanings & $\begin{array}{l}\text { The rational, symbolic and } \\
\text { affective associations of } \\
\text { particular actions }\end{array}$ & $\begin{array}{l}\text { Understanding of what meals } \\
\text { are; association of foods with } \\
\text { identity, family, history or place }\end{array}$ \\
\hline Competences & $\begin{array}{l}\text { Practical knowledge of how to } \\
\text { perform actions, and } \\
\text { propositional knowledge about } \\
\text { the actions }\end{array}$ & $\begin{array}{l}\text { Ability to cook; knowledge of } \\
\text { where to procure ingredients }\end{array}$ \\
\hline
\end{tabular}


Immigration to Vancouver from Europe, China and elsewhere has continued in waves since then, despite anti-Chinese laws and policies being implemented in the early twentieth century. Since the liberalisation of immigration laws in the 1960s and 1970s, the population and consequently the food environments within the city have rapidly diversified (Gibb \& Wittman, 2013; Statistics Canada, 2017). In the late twentieth century, waves of immigrants arrived in Vancouver, particularly from a number of South Asian and Southeast Asian countries, including India, the Philippines and Vietnam. By the 2016 national census, nearly $45 \%$ of the city's population were born outside Canada (first generation) with an additional $25 \%$ having at least one parent born outside Canada (second generation; Statistics Canada). The largest population groups in Vancouver (as categorised by Statistics Canada) are European, Chinese, South Asian, Filipino and Korean.

Both the long multicultural history and the more recent diversification of food environments made Vancouver an ideal site for this study. Many of the residents interviewed had witnessed some degree of change in the availability of ethnic foods in Vancouver over their lifetime and were able to reflect on the extent to which this had influenced their diets and the diets of other generations in their families.

\section{Methods}

\subsection{Participants}

This paper is based on data collected through interviews and participant observation with adult participants in Vancouver, Canada in 2018. Participants who self-identified as either Chinese-descent or European-descent were selected for this study as representative of Asian Canadians and White Canadian racial categories. Both of these racial categories include people of multiple ethnicities. The authors acknowledge that race and ethnicity are social constructs that have cultural implications related to food practices, and both concepts are limited as categories for analysis. The decision to use race as an identifier for participants in this research was informed by previous work in Vancouver (Gibb \& Wittman, 2013) and elsewhere (Flowers \& Swan, 2016) that elucidates how race as an axis of inequality has impacted the structure of local food environments historically, and continues to mediate how communities interact with their food environments. Chinese- and White European-Canadians were selected because they form the greatest proportion of Vancouver's population and because both groups have had long-standing impacts on the food system and food environments of the city.

Sixteen participants took part in semi-structured interviews. Participants ranged in age from 19 to 70 and in generation status from those born outside Canada (first generation), to those whose families had lived in Canada for more than 100 years. All participants were responsible for their own food provisioning and dietary choices. Participants were recruited using an online survey that requested basic demographic information, and asked participants about how long and where in Vancouver they had lived, and about how often they bought and prepared their own food. The survey was distributed through academic and civil society networks. In addition to the survey, purposeful sampling through participants' networks was used to increase the age range and race and gender balance of participants. More participants were female than male, which may have resulted partially from disproportionate female interest and participation in food culture, and from recruitment through gendered community networks. Ethical approval for the study was obtained through the University of Oxford's Central University Research Ethics Committee (ref: SOGE-18A-110) and all participants gave written or oral (where telephonic interviews were conducted) prior informed consent to participate in the study.

\subsection{Interviews}

A semi-structured approach to interviews allowed the interviewer flexibility to respond to participants' cues and to reduce the potential for closed questions that could be perceived as normative and thereby bias participants' responses. The interviews lasted between 20 and $45 \mathrm{~min}$. Participants chose the interview venue and were given the option of doing a walk-and-talk interview. Five participants opted for a walk-andtalk interview and showed the interviewer around the shops, restaurants and/or gardens where they obtained their food while being interviewed. The walk-and-talk interviews presented opportunities for the observation of food environments and shopping practices and to ask participants questions based on observed cues. Two participants were interviewed telephonically. The interview questions related to participants' eating habits, food choices, food environments and observations of their friends' and families' eating habits. An outline of the interview questions is provided in Appendix I. All interviews were digitally recorded.

\subsection{Participant and unstructured observation}

Participant observation was used opportunistically to obtain data regarding food environments, shopping and learning-to-cook practices of Vancouver's residents. Opportunities for participant observation arose based on the development of trust between the researcher and participants and the willingness of the latter to involve the researcher in local food practices. These opportunities included a community cooking workshop attended with interview participants by one of the authors, and participant observation of shopping, food gardening, and public food practices with interview participants across Vancouver. In addition, unstructured observation of the participants' food environments was conducted in large supermarkets, small independent greengrocers, ethnic food stores, farmers' markets, and ethnic food restaurants. Locations for observation were selected based on interview participants' descriptions and recommendations, and included parts of Vancouver that participants frequented and those that are known for the availability of specific cuisines and ingredients, for example Chinatown. Detailed observations were recorded in field notes. These observational data were used to validate interview data relating to food environments and provided the researchers with a deeper contextual understanding of how food environments (including media and advertising in shops and restaurants) contributed participants' understanding of the environmental and health implications of their dietary choices which they referred to in interviews. They also provided insights into cross-cultural sharing of food knowledge through the community cooking workshop and observations of popular ethnic food retailers, restaurants and neighbourhoods.

\subsection{Data analysis}

All interviews were conducted and observational data collected by the lead author, who also conducted the initial data analysis. Interviews were summarised in detail immediately after the interview. Key passages from each interview were transcribed iteratively as themes emerged from the coding. All data included in the summaries, transcriptions and field notes were analysed using a combined inductive and deductive approach to integrating theory and data. Initially, codes were developed inductively from the data and grouped thematically (for example, "interpersonal relationships", "changes in the food environment", "home food"), following a thematic analysis approach (Guest et al., 2011). Then, the social practice theory framework (Shove et al., 2012; Twine, 2018) was used to identify three domains of practice and their constituent elements. This was done by analysing data coded for each element of practice (materials, meanings and competences) and identifying commonly occurring relationships among elements. The relationships between the domains and processes of change within them were analysed using codes that indicated when participants spoke about changes in their diets.

The data were coded iteratively as codes and themes were developed, and additional passages were identified for more detailed coding. 
During this process, the authors collaborated to assess and interpret the codes. After the initial analysis, the data were examined for theoretical congruence with existing literature and theories of dietary change. Block quotes are presented below to aid interpretation and increase transparency of the analytical process. NVivo software was used to facilitate the coding and data analysis.

\section{Results}

Of the 16 participants, 13 observed an increased variety of foods in their diets, which they explicitly linked to the availability of diverse foods in their food environments. For example, several participants observed that their diets had changed to incorporate new foods when they moved to Vancouver or to a different part of Vancouver, because those foods were easily available in their new food environment. Seven of these participants also linked the diversification of their diets to interactions with friends and neighbours from different ethnic groups to themselves. New foods associated with cuisines of other ethnic groups were often tasted at restaurants or in friends' homes and gradually incorporated into their diets. Although the trend of dietary diversification was common, the extent to which participants adopted new foods and food practices and adapted their old ones varied between participants and practices. For some participants, only the food they consumed in restaurants became more diverse, while for others, the foods they cooked at home, ate in public, and used to celebrate special occasions all became more diverse. Identifying the three domains of practice (Section 1.1.), common to all participants, illuminated the nuances of this trend of diversification.

Practices most commonly fell into the domain of everyday food practice. This domain included the practices performed by participants to address their daily need to eat and be nourished, often involving routines and habits. In contrast, public food practices were those performed outside of daily routines, including eating at restaurants and dinner parties, often associated with social gatherings. Finally, festive food practices were performed on special occasions, including religious and cultural holidays. Meals and food preparation in the festive food domain typically involved larger groups of people than everyday food practices and were associated with intergenerational relationships and interpretation of traditions and cultures.

An explanation is given below of how food practices in each of the three domains changed through the process of dietary acculturation (3.1). This description focuses on how meanings and competences mediated the change in materials in each domain. An explanation of the role that interpersonal relationships played in facilitating dietary acculturation (3.2), and patterns in the interactions between traditions, generational change and dietary acculturation (3.3) based on the data are also presented.

\subsection{Processes of dietary acculturation and domains of practice}

\subsubsection{Public food practices}

For almost all participants, dietary change - through the addition of new foods into their diets - happened most frequently in the public food domain, where practices included eating at restaurants and dinner parties. Several participants observed that more restaurants, offering more diverse cuisines had opened in their neighbourhoods and elsewhere in the city over the last two decades. This growing diversity of restaurants in Vancouver (Cheung, 2018; Rule, 2018) presented opportunities for residents to taste and incorporate new foods (materials) into their diets and food practices. Several participants associated public food practices with meanings like novelty or variety as well as with pleasure.

"When I go out, I like to eat stuff that I usually don't make at home." (Chinese-Canadian, 30-39, female)
“[The food I like to eat when I go out] I guess is stuff I can't make at home, like vegan junk food, like burgers and fries." (Chinese-Canadian, 19-29, female)

Linked to this association with novelty was a willingness to take risks and eat unfamiliar foods that was more commonly practiced in this domain than in the other two domains. As a result, public food practices were the sites where participants most frequently tasted new foods.

"When I look back to other things, like when I have tried other foods, it's usually because I have had the opportunity to have it cooked for me." (European-Canadian, 50-59, female)

Some foods tasted under these circumstances became frequent materials in participants' public food practices, but the transfer of these foods into everyday and festive food practices was less common and usually happened over an extended time period.

\subsubsection{Everyday food practices}

For most participants, dietary change in the everyday food domain happened less frequently and over a longer period than in the public food domain. Practices in this domain often became habitual, like grocery shopping at the same time and place every week or eating meals every day in the same place with the same people.

Several factors constrained changes in everyday food practices, relating to meanings and competences that were characteristic of this domain. The difficulty or energy required to change habits and routines was one such constraint (Williams-Forson, 2014). Another was the emotional meanings relating to everyday food practices. For example, several participants spoke about childhood memories and how emotional associations of the food practices they grew up with influenced the meanings of their everyday food practices as adults (Williams-Forson, 2014).

"After a period of time, I was like, 'You know what, I can't keep eating this way.' And I went back to the diet I grew up on." (European-Canadian, 40-49, female)

Even when participants identified changes to their everyday food practices as desirable, they sometimes felt limited by the competences required to make these changes. One participant identified the time available for preparing meals as a limitation on incorporating new ingredients into everyday food practices.

"For our family, we eat a whole variety of cultural-influenced foods, but it's not something that's homemade. And that's just a time constraint." (European-Canadian, 40-49, female)

The ability to cook was a commonly identified competence that influenced dietary change in everyday food practices. Although it was mentioned by a few participants in relation to hosting dinner parties (a public food practice) or preparing celebratory meals (a festive food practice), cooking was a competence particularly associated with everyday food practices. It was one of the most influential elements in determining how the extent and speed of dietary change differed between domains of practice and among participants.

A few participants identified their limited cooking know-how as a constraint on the materials or ingredients they used in everyday food practices.

"For a semester I was trying to go to farmers' markets and buying stuff there, but it was really challenging ... Also the vegetables, I didn't know how to cook some of them." (Chinese-Canadian, 19-29, female)

This particularly limited the ability of some participants to transfer new foods from their public food practices into their everyday food practices. 
"I always think that [I should try making that restaurant food at home], but I never end up doing it. I always just resort back to the easy things that I know how to make ... I feel like I don't have enough time and also I don't know if I'm good enough of a chef to recreate it." (Chinese-Canadian, 19-29, female)

In contrast, other participants saw their ability and confidence with cooking as an opportunity to diversify their diets and incorporate foods from their public food practices into the everyday domain. For these participants, culinary acculturation - the process through which participants adopted cooking and food preparation methods typical of other cultures - facilitated a dietary transition in the everyday food domain (Kalyoncu, 2018; Li, 2020).

"But I think a lot of it comes from just practice. You start with your macaroni and cheese kind of thing as a young person or your noodles. And then you realise that ground beef is pretty easy to cook. But now, I'm not afraid to cook anything." (European-Canadian, 50-59, male)

"I found that I really liked butter chicken and a bean and chickpea dish, I really like that. So one year I asked for an Indian cookbook, so now I just make it at home. I've tried them at restaurants and I've brought them home." (European-Canadian, 40-49, female)

Dietary acculturation in the everyday food domain was often dependent on dietary change in the public food domain, where participants were most frequently introduced to foods from other ethnic groups and became accustomed to them. Processes of learning new cooking methods and to cook new foods - particularly from friends and family members - were pivotal in enabling this change.

\subsubsection{Festive food practices}

Practices in this domain were performed occasionally and associated with celebrations or rites of passage. Most commonly, participants spoke about engaging in these practices on cultural holidays like Christmas, Easter and Thanksgiving (mostly European-Canadian participants) or Lunar New Year and the Harvest Moon Festival (mostly ChineseCanadian participants). The food practices they engaged in on these occasions were linked to the meanings of the festivals and often continued almost unchanged from year to year. These practices were also often associated with extended families, family traditions, and intergenerational relationships. Participants spoke about inheriting traditional food practices, like making dumplings at Lunar New Year or turkey at Thanksgiving, from their parents and grandparents. Even participants who felt that their diets had largely departed from what their families traditionally ate said festive food practices were a time when they returned to their traditional foods.

"My Polish background really ends up being at holidays or just to keep a little tradition for the kids ... Traditionally, the Polish foods that we had were mostly, I remember, at festival times such as Christmas, Easter." (European-Canadian, 60-69, male)

Although continuity was frequently mentioned in relation to festive food practices, some significant changes were described. One of these was acculturation relating to the adoption or celebration of new festivals. For example, some Chinese-Canadian participants spoke about celebrating Christmas or Thanksgiving as something they had started to do because others around them did it, even though it had not been part of their cultural heritage.

“We celebrate Christmas, but it's very superficial. We're not Christian or anything, but it's just an opportunity for all our family to get together. And Thanksgiving kind of the same ... We would occasionally have turkey, but it was really only because everyone else was having turkey and that's the thing to do." (Chinese-Canadian, 30-39, female)

Some participants also described how festive food practices changed between generations. Specifically, younger participants reported that who they celebrated festivals with and how they celebrated them was changing. These young people noted that they now celebrated Christmas, Thanksgiving or Lunar New Year with their friends, instead of, or as well as, with their families. In these situations, and in some family settings, the younger generation became the carriers of festive food practices and changed them to suit their own meanings and competences. Two young Chinese-Canadian participants describe their experiences of this:

"I think as the grandkids, like my cousins, as we got older and when we learned how to cook, we would cook stuff that was not always Asian. One of my cousins, he really likes cooking. I remember one Christmas he cooked a Persian dish, Persian stew." (Chinese-Canadian, 30-39, female)

"I just do them [celebrate Lunar New Year and Harvest Moon Festival] with my friends. And when I do them with my friends, it's a lot more customised to everyone's tastes. So people have more control over what's in the ingredients and that kind of thing." (Chinese-Canadian, 20-29, female)

In these examples, young Chinese-Canadians used their own competences (abilities to combine ingredients and cook dishes) to change their festive food practices by changing the materials (ingredients) and competences (cooking methods) they used, and so influencing the experiences (meanings) they and others associated with these festive food practices. These changes in festive food practices often reflected culinary acculturation and dietary change that had taken place in the everyday and public food domains.

\subsection{Cross-cultural relationships}

Changes in food environments increased the range of available materials that could be incorporated into the food practices of Vancouver's residents. However, the change in materials alone was not sufficient to drive dietary acculturation. Changes in the meanings (for example taste, healthiness and familiarity) associated with new materials and the competences required to procure and prepare foods were also a necessary component of dietary change. In all three domains, participants' cross-cultural relationships played an important role in introducing diverse foods and driving the change in meanings and competences.

Participants reported that they often tried things at restaurants for the first time on the recommendation of friends and family who were familiar with those foods. In some cases, as the quote below describes, new foods were also introduced in the homes of their friends from other cultures.

"For Korean food, one of my best friends from elementary school, she's from Korea. She moved here when she was like ten. I actually got introduced to Korean food at her house. Her mom would make us some food." (European-Canadian, 30-39, female)

If they liked the foods they were introduced to in this way (positive meanings), participants would often continue to eat them in restaurants. Some participants also learned to cook new foods from friends and neighbours of other ethnic groups, like one participant who learned to make fresh pasta from an Italian-Canadian neighbour. In this way, they developed competences that allowed them to incorporate new foods into their everyday food practices. However, foods were not often transferred from the public food domain into the everyday food domain, because participants mostly lacked the competences to prepare and cook the new foods themselves.

Cross-cultural sharing of food practices also permeated the festive food domain. This was particularly true for young participants with multicultural friend groups or for participants whose families spanned ethnic groups. For example, one European-Canadian participant had a Chinese-Canadian son-in-law and reported learning about and 
participating in Lunar New Year practices and other Chinese-Canadian traditions with her son-in-law's family. Another Chinese-Canadian participant reported adopting some of the festive food practices of her British husband and sharing her own practices with him.

\subsection{Tradition and generational change}

Almost all participants across age groups reported that the younger generations in their families had more diverse diets and were more willing to try new foods than the older generations, suggesting that dietary acculturation was visible on a generational time scale. The willingness to diversify diets was linked to meanings like familiarity, taste preferences and ideas about what constitutes a meal.

"A couple of years ago, my mother tried pizza for the first time, and that was a bit of a struggle for her. It's cheesy, and that's not a flavour she can wrap around her head. She'll stick with typical noodles and soup ... She'll prefer to stick with the soups and tofu and stuff that she's more familiar with." (Chinese-Canadian, 40-49, female)

For the European-Canadian participants, dietary changes were often characterised by a move away from a meal format with separate portions of meat, potatoes and vegetables (Crowther, 2013). Both Chinese- and European-Canadian participants reported eating more foods from other cuisines than older family members. These generational differences were evident to participants from practices in all three domains, but were most noticeable in festive food practices where intergenerational family relationships played a significant role (see 3.1). In these practices, people of different generations carried, interpreted and performed traditions in different ways, making festive occasions into microcosms of societal patterns of cultural change (Geertz, 1957).

A contrasting perspective on generational dietary acculturation was presented by a few young Chinese-Canadian participants. These participants suggested that their generation associated more positive meanings and valued the continuation and transmission of their traditional Chinese food practices more highly than some people of older generations in their families. These participants felt that their parents and grandparents were promoting dietary acculturation of the younger generation - specifically the adoption of dominant (mostly EuropeanCanadian) food practices - as a way to integrate into society. These participants expressed a sense of loss that they had not learned the competences required for some traditional Chinese food practices:

"Our elders are pushing us to conform ... The next gen[eration] is just like, 'Wait. When I move out where is the dishes that I grew up on that makes me feel at home?'" (Chinese-Canadian, 30-39, male)

The meanings that these young Chinese-Canadians associated with their traditional food practices differed from those of their ChineseCanadian elders who, they reported, thought that traditional Chinese foods were no longer appropriate to their context. In contrast, the young Chinese-Canadians saw value in these traditional practices and sought to reconnect with them:

"I know for myself and my peers, they want to eat more of the traditional foods, but they don't know where to find it, or there aren't any restaurants that have it, or they don't know how to cook it anymore. Because they didn't learn that, the recipes have not been passed down. That can be a bit concerning as well, because all those traditional, it's part of the culture and it's slowly dying." (ChineseCanadian, 30-39, female)

\section{Discussion}

This research presents evidence for dietary change among residents of Vancouver, where the food environments and population are diversifying as a result of immigration. By illustrating changes in everyday, public, and festive food practices, the results show dietary acculturation in process among Chinese- and European-Canadians. This finding offers insight into dietary acculturation as an ongoing, multi-directional process that influences dietary transitions of the largest population groups in Vancouver. In this way, the results broaden our understanding of the relationship between cultural interactions and dietary change, as well as opening further questions about the roles of festivals, and intergenerational and cross-cultural relationships in dietary transitions.

Based on these findings, we have developed an exploratory model of the stages of dietary acculturation for majority or large minority population groups in a cosmopolitan urban context. This model expands on the existing literature on processes of acculturation and dietary change. The purpose of this model is to offer a heuristic for understanding dietary acculturation in process and a framework for identifying further questions about how acculturation relates to dietary transitions. The stages identified correspond to the processes of dietary change observed in the three domains of practice (Fig. 1). In the first stage, acculturation occurs through the addition of new foods into public food practices. In the second stage, new foods are integrated into everyday food practices. Finally, new or hybrid (combined new and old) meal components or elements replace old ones in festive food practices.

The identification of these three stages is supported by Rothman et al. (2009) review of dietary change literature, where the initiation of dietary change (for example through the addition of new foods) is identified as a separate stage of the process to the maintenance of dietary change (for example through the integration of new ingredients into cooking practices). Similarly, the addition of new foods into diets and the exclusion or replacement of traditional foods are also identified as two distinct processes in studies on dietary change (Tu et al., 2012). Analogous to the stages we have identified, Bickerton (1984) describes three stages in the development of new hybrid languages in his work on linguistic acculturation. He argues that the changes from a pidgin (hybrid of two or more languages with no formal grammar) into a creole (with a structured grammar), and finally into a new language happens as successive generations learn to speak. Likewise, in this study, older generations were less likely to move beyond the first stage of dietary acculturation than their children and grandchildren, and it was young adults (18-30) - who were relatively new to cooking — who most frequently engaged in replacing old components of festive foods with new ones. In this way, over generational time, new and hybrid foods and foodways become part of the 'cultural grammar' of these communities. Despite this analogy, we do not propose that these stages are biologically driven or happen linearly, but rather that they are overlapping and iterative.

Acculturation through addition in the public food domain was the most common form of dietary acculturation among participants in this research. This involved the addition of new foods into participants' diets, which happened most frequently at restaurants where they interacted with new foods in the form of composed meals (rather than interacting with the individual ingredients). In the public food domain, participants engaged with their changing and diversifying food environments through consumption (Flowers \& Swan, 2012). This process of seeking and experiencing novelty and diversity by eating new foods and cuisines is identified by Flowers and Swan (2012) as 'food adventuring' and is linked to the formation and articulation of identities. Consuming diversity can be part of how people develop and articulate their identities as urban cosmopolitan citizens (Jonas, 2013; Shaw et al., 2004). In this way the meanings of public food practices are linked to the media framing of 'global cities' as cosmopolitan destinations where ethnic diversity can be explored and consumed in the form of food - "eating the Other" (Shaw et al., 2004; hooks, 1992 quoted in; Flowers \& Swan, 2012). Similarly, according to Chapman and Beagan (2013), multiple transnational identities arise in food practices reflective of different cultures. For example, in our study, food adventuring and identity formation in the public domain was, in some cases, contrasted with conservatism in the other domains of practice. 


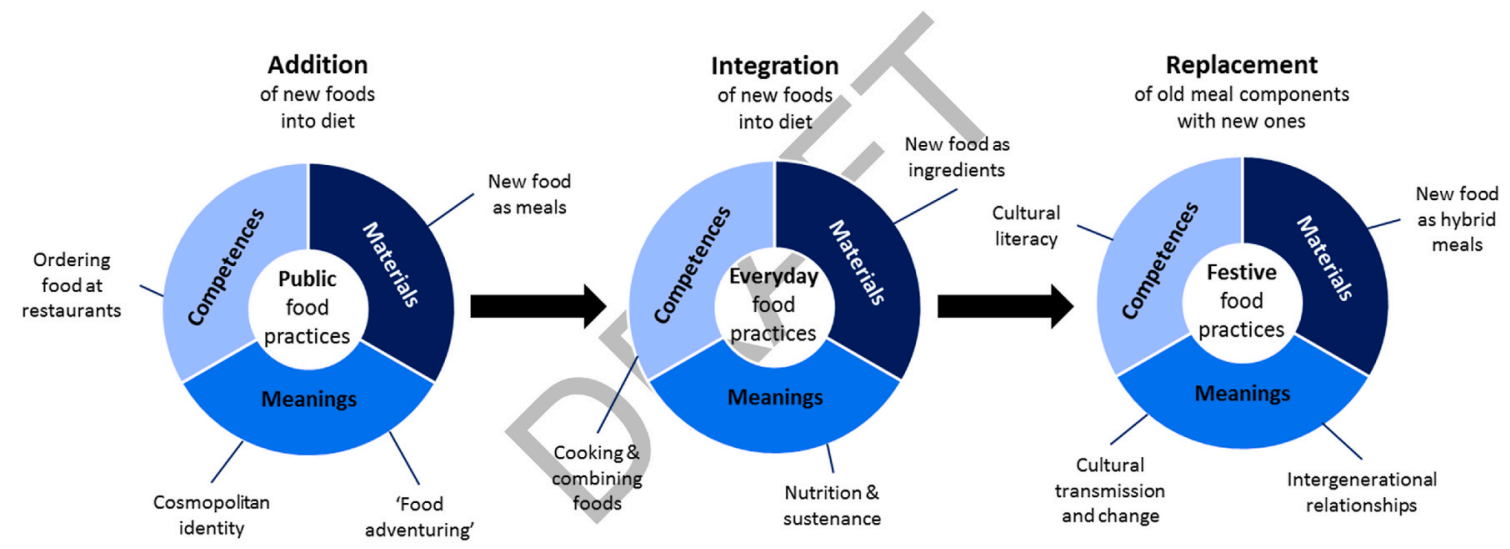

Fig. 1. Three stages of dietary acculturation - Addition, Integration and Replacement - and their associated changes in materials, meanings and competences.

"We do this English-speaking and sandwich-eating thing outside, publically, but we go home and it's traditional as hell." (ChineseCanadian, 30-39, male)

As explored in Section 3.1, changing practices within the public food domain requires relatively few or easily-acquired new competences. For example, an assumed familiarity with ethnic cuisines and the associated language of "vernacular foodways" (Jonas, 2013) as they are presented in ethnic restaurants, contributed to this stage of dietary acculturation (Flowers \& Swan, 2012).

The second stage of dietary acculturation proposed is the integration of new foods into everyday food practices such as the substitution of ingredients (Williams-Forson, 2014). In contrast to the first stage, new foods in this stage were usually in the form of ingredients and were integrated into existing everyday food practices rather than being added to the repertoire of pre-composed meals. This required a more advanced set of competences than ordering off a menu, including identifying, combining and preparing new ingredients. For example, one European-Canadian participant learned to prepare sushi at home, and through this process changed from engaging with sushi in its pre-composed form to buying, preparing, and combining fish, rice, seaweed, and other materials. This example illustrates how culinary acculturation facilitated dietary change in the everyday food domain. At this stage of acculturation, new foods take on similar meanings to other everyday food practices, becoming familiar and considered for their nutritional value and sustenance (Crowther, 2013; Flowers \& Swan, 2012). Through contact with other cultures, the everyday food practices of Chinese- and European-Canadians began to incorporate foods from other ethnic groups in Vancouver - the second stage of dietary acculturation.

In the third stage of dietary acculturation, components of traditional meals in festive food practices were replaced with new or hybrid components containing new ingredients. This form of acculturation was less commonly observed than the first two stages. It was mostly described by younger participants (19-30) in this study, or referred to by participants in relation to the younger generations in their families. For example, younger generations prepared non-traditional dishes for family gatherings at Christmas, Lunar New Year or Thanksgiving. In addition to requiring competences linked to combining ingredients and preparing foods, acculturation in the festive food domain required these young people to be culturally literate. They performed traditional food practices, often inherited from their parents and grandparents, and deliberately changed those practices to include new or hybrid foods once the responsibility for carrying these traditions became theirs. Analogous to new languages in Bickerton's (1984) work on linguistic acculturation, it is possible that these new festive food practices would be transmitted to future generations not as a hybrid product of acculturation, but as persistent traditions or components of the culture. In this way, and over a generational timescale, contact with other cultures influences the food practices that are considered to be traditional for Chinese- and European-Canadians.

It was outside the scope of this research to study historical dietary change beyond that which the research participants could recall. It is likely, however, that in Vancouver as in other places, dietary acculturation has been ongoing at least since the area was settled by British and Chinese people and that what are currently thought of as authentic Chinese- or European-Canadian practices have changed through acculturation over generations (Crowther, 2013).

While the processes of population and food environment diversification in Vancouver are paralleled in many cities in North America and Europe (Thomas, 2006), we acknowledge that the stages of dietary acculturation identified in this study may differ in some aspects to the process in other cities. It is possible that the rate of dietary acculturation for majority and large minority population groups is faster in Vancouver than in many other cities as a result of the scale of immigration and speed at which the population and food environments have diversified. In addition, literature suggests that processes of dietary change and acculturation follow a different course for people in small ethnic minorities and with lower incomes (Chapman \& Beagan, 2013; Miewald \& McCann, 2014).

Despite these limitations, this research contributes to our understanding of dietary dynamics in a modern urban context. First, it contributes a methodological approach for exploring cultural transitions that prioritises deep description and contextualisation, by using mixed data collection methods and an analytical framework based on social practice theory. Second, the exploratory model above presents a framework for developing lines of enquiry into how cultural interactions affect dietary transitions. It would be interesting to understand the extent to which the stages of acculturation suggested reflect the processes of dietary acculturation in other cities and among other ethnic groups. This model can also be used to generate questions and hypotheses about how materials, meanings and competences change and interact through dietary transitions, and to explore the roles of ritual and intergenerational relationships in these transitions.

Dietary practices, particularly in cosmopolitan urban contexts, are constantly in flux, as diverse ethnic groups come into contact, and new generations develop their own hybrid food cultures. This understanding of dietary change is relevant to the design of increasingly common interventions aiming to facilitate a transition to healthier and more sustainable diets. Such dietary change interventions frequently consider cultural acceptability in their design, but often do not go beyond the recognition that different groups eat different foods (Crowther, 2013; Hammelman \& Hayes-Conroy, 2015; Wang, 2011). A focus on food practices, incorporating insights on meanings and competences, could move such interventions towards more effectively addressing drivers of dietary change (Hammelman \& Hayes-Conroy, 2015). Similarly, an 
understanding of diversity-in-process could be used to link these dietary interventions with ongoing dietary change. For example, this could be done by developing the competences (like cooking skills) needed to integrate new foods into everyday practices, or offering education about ethnic foods that links them to meanings associated with everyday food practices like health, sustenance or sustainability, instead of only novelty and pleasure. This research expands our understanding of the links between cultural change and dietary transitions, which, when drawn into the planning and practice of dietary change interventions, offers prospects for more sustainable and inclusive outcomes.

\section{Author contributions}

Nicola Frances Rule was responsible for the conception and design of the study, data collection, data analysis and interpretation, and drafting the article and contributed to the critical revision of the article. Colin Charles Dring provided support for the design of the study and contributed to the interpretation of the data and critical revision of the article. Thomas F. Thornton contributed to the interpretation of the data and critical revision of the article. All three authors provided final approval of the version to be published.

\section{Funding sources}

The field work was kindly funded by the Environmental Change Institute and Pembroke College, University of Oxford. None of the funding sources for this research were involved in study design, in the collection, analysis and interpretation of data, in the writing of the report, or in the decision to submit the article for publication.

\section{Data availability}

The lead author has full access to all the data reported in this manuscript.

\section{Ethics statement}

Ethical approval for the study was obtained through the University of Oxford's Central University Research Ethics Committee (ref: SOGE-18A110 ) and all participants gave written or oral (where telephonic interviews were conducted) prior informed consent to participate in the study.

\section{Acknowledgements}

The authors would like to thank Dr Monika Zurek, Dr Sarah Darby, Dr Ariell Ahearn and Bernard Soubry for their conceptual contributions to this work. We are also grateful to the sixteen research participants who generously shared their wisdom and experience with us, providing a wealth of data for this study. This research was conducted as part of the lead author's MSc in Environmental Change and Management at the University of Oxford, which was undertaken with the support of a Commonwealth Scholarship, funded by the British government.

\section{Appendix A. Supplementary data}

Supplementary data to this article can be found online at https://doi. org/10.1016/j.appet.2021.105728.

\section{References}

hooks, b (1992). Eating the other. In Black looks: Race and representation (pp. 21-39). Boston: South End Press.

Ayala, G. X., Baquero, B., \& Klinger, S. (2008). A systematic review of the relationship between acculturation and diet among latinos in the United States: Implications for future research. Journal of the American Dietetic Association, 108, 1330-1344. https:// doi.org/10.1016/j.jada.2008.05.009
Berkes, F. (2012). Sacred ecology (3rd ed.). Philadelphia: Taylor and Francis.

Bickerton, D. (1984). The language bioprogram hypothesis. Behavioural and Brain Sciences, 7(2), 173-221.

de Boer, J., \& Aiking, H. (2017). Pursuing a low meat diet to improve both health and sustainability: How can we use the frames that shape our meals? Ecological Economics, 142, 238-248. https://doi.org/10.1016/j.ecolecon.2017.06.037

Chapman, G. E., \& Beagan, B. L. (2013). Food practices and transnational identities: Case studies of two Punjabi-Canadian families. Food, Culture and Society, 16(3), 367-386. https://doi.org/10.2752/175174413X13673466711688

Chen, X., \& Kwan, M.-P. (2015). Contextual uncertainties, human mobility, and perceived food environment: The uncertain geographic context problem in food access research. American Journal of Public Health, 105(9), 1734-1737. https://doi. org/10.2105/AJPH.2015.302792

Cheung, C. (2018, April 23). The deliciously diverse food of 1.6 billion Chinese comes to vancouver. The Tyee. Retrieved from https://thetyee.ca/News/2018/04/23/Delici ous-Diversity-Billion-Chinese-Food-Vancouver/.

Conijn, J. G., Bindraban, P. S., Schröder, J. J., \& Jongschaap, R. E. E. (2018). Can our global food system meet food demand within planetary boundaries? Agriculture, Ecosystems \& Environment, 251, 244-256. https://doi.org/10.1016/j. agee.2017.06.001

Crowther, G. (2013). Eating culture. Toronto: University of Toronto Press.

Das Gupta, M. (1997). "What is Indian about you?": A gendered, transnational approach to ethnicity. Gender \& Society, 11, 572-596.

Flowers, R., \& Swan, E. (2012). Eating the Asian other? Pedagogies of food multiculturalism in Australia. Portal Journal of Multidisciplinary International Studies, 9(2), 1-30. https://doi.org/10.5130/portal.v9i2.2370

Flowers, R., \& Swan, E. (2016). Potatoes in the rice Cooker: Family food pedagogies, bodily memories, meal-time senses and racial practices. Food Pedagogies (Ashgate Publishing).

Geertz, C. (1957). Ritual and social change: A Javanese example. American Anthropologist, 59, 32-54.

Gibb, N., \& Wittman, H. (2013). Parallel alternatives: Chinese-Canadian farmers and the metro vancouver local food movement. Local Environment, 18(1), 1-19. https://doi. org/10.1080/13549839.2012.714763

Greene, G. W., Rossi, S. R., Rossi, J. S., Fava, J. L., \& Prochaska, J. O. (1999). Dietary applications of the stages of change model. Journal of the American Dietetic Association, 99(6), 673-678.

Guest, G., MacQueen, K., \& Namey, E. (2011). Introduction to Applied thematic analysis. In Applied thematic analysis (pp. 3-20). New York: SAGE Publications. https://doi. org/10.4135/9781483384436.n1.

Hallowell, A. I. (1959). The impact of the American Indian on American culture. American Anthropologist, 59, 201-217.

Hammelman, C., \& Hayes-Conroy, A. (2015). Understanding cultural acceptability for urban food policy. Journal of Planning Literature, 30(1), 37-48. https://doi.org/ $10.1177 / 0885412214555433$

Holland, K. M. (2007). A history of Chinese immigration in the United States and Canada. American Review of Canadian Studies, 37(2), 150-160. https://doi.org/10.1080/ 02722010709481851

Huang, Q. (2019). Drinking tea in st. John's: A study of diasporic Chinese tea drinking and ethnic identity. Masters thesis. Memorial University of Newfoundland.

Jonas, T. (2013). Eating the vernacular, being cosmopolitan. Cultural Studies Review, 19 (1), 117-137. https://doi.org/10.5130/csr.v19i1.3076

Kalyoncu, Z. B. (2018). Development of a new scale to measure Culinary Acculturation of immigrants who are living in Turkey: Validity and reliability assessment. $\mathrm{PhD}$ thesis. Hacettepe University.

Li, M. (2020). Performative Chineseness and culinary tourism in Chinese restaurants in Newfoundland, Canada. Folklore, 131(3), 268-291. https://doi.org/10.1080/ 0015587X.2019.1703416

Miewald, C., \& McCann, E. (2014). Foodscapes and the geographies of poverty: Sustenance, strategy, and politics in an urban neighborhood. Antipode, 46(2), 537-556. https://doi.org/10.1111/anti.12057

Migration Data Portal. (2021). World key migration Statistics. Retrieved February 20, 2021, from https://migrationdataportal.org/? $\mathrm{i}=$ stock_abs_\& $\mathrm{t}=2020$.

National Collaborating Centre for Environmental Health NCCEH. (2017). Food environments. Retrieved August 18, 2018, from http://www.ncceh.ca/environ mental-health-in-canada/health-agency-projects/food-environments.

Orgnaisation for Economic Cooperation and Development OECD. (2019). International migration and displacement trends and policies: Report to the G20. Retrieved from https://www.oecd.org/migration/mig/G20-migration-and-displacement-trends-and -policies-report-2019.pdf.

Poore, J., \& Nemecek, T. (2018). Reducing food's environmental impacts through producers and consumers. Science, 360, 987-992.

Popkin, B. M. (2006). Global nutrition dynamics: The world is shifting rapidly toward a diet linked with noncommunicable diseases. American Journal of Clinical Nutrition, 84, 289-298. https://doi.org/10.1093/ajcn/84.2.289 [pii].

Rothman, A. J., Sheeran, P., \& Wood, W. (2009). Reflective and automatic processes in the initiation and maintenance of dietary change. Annals of Behavioral Medicine, 38 (Suppl.1), 4-17. https://doi.org/10.1007/s12160-009-9118-3

Rouse, R. (1995). Questions of identity: Personhood and Collectivity in transnational migration to the United States. Critique of Anthropology, 15, 351-380.

Rule, N. F. (2018). Meals in the melting-pot: The role of immigration and cultural diversity in sustainablt dietary transitions in Vancouver, Canada. Masters thesis. University of Oxford.

Sanou, D., O’Reilly, E., Ngnie-Teta, I., Batal, M., Mondain, N., Andrew, C., ... Bourgeault, I. L. (2014). Acculturation and nutritional health of immigrants in 
Canada: A scoping review. Journal of Immigrant and Minority Health, 16, 24-34. https://doi.org/10.1007/s10903-013-9823-7

Satia-Abouta, J., Patterson, R. E., Kristal, A. R., The, C., \& Tu, S. P. (2002). Psychosocial predictors of diet and acculturation in Chinese American and Chinese Canadian women. Ethnicity and Health, 7, 21-39.

Schatzki, T. R. (1996). Social practices: A wittgensteinian approach to human activity and the social. Cambridge, UK: Cambridge University Press.

Shaw, S., Bagwell, S., \& Karmowska, J. (2004). Ethnoscapes as spectacle: Reimaging multicultural districts as new destinations for leisure and tourism consumption. Urban Studies, 41(10), 1983-2000. https://doi.org/10.1080/ 0042098042000256341

Shove, E., Pantzar, M., \& Watson, M. (2012). The dynamics of social practice: Everyday life and how it changes. London: Sage.

Steffen, W., Richardson, K., Rockstrom, J., Cornell, S. E., Fetzer, I., Bennett, E. M., ... Sorlin, S. (2015). Planetary boundaries: Guiding human development on a changing planet. Science, 347(6223). https://doi.org/10.1126/science.aaa9629, $1259855-1-10$

Thomas, R. (2006). The impacts of neighbourhood food choices and acculturation on immigrant diets. Plan 581: Non-Motorized transport. University of British Columbia.
Tu, V. P., Husson, F., Sutan, A., Ha, D. T., \& Valentin, D. (2012). For me the taste of soy is not a barrier to its consumption. And how about you? Appetite, 58, 914-921. https:// doi.org/10.1016/j.appet.2012.01.023

Twine, R. (2018). Materially constituting a sustainable food transition: The case of vegan eating practice. Sociology, 52(1), 166-181. https://doi.org/10.1177/ 0038038517726647

Vallianatos, H., \& Raine, K. (2008). Consuming food and constructing identities among Arabic and South Asian immigrant women. Food, Culture and Society, 11, 355-373.

Wang, M. (2011). Multicultural engagement on sustainability: Engaging the Chinese community on the greenest city action plan. University of British Columbia.

Williams-Forson, P. (2014). "I haven't eaten if I don't have my soup and fufu": Cultural Preservation through Food and Foodways among Ghanaian Migrants in the United States. Africa Today, 61(1), 69-87.

Ye, T. (2018). Somewhere in between: Chinese identity and community in st. John's as seen through Chinese restaurants. Masters thesis. Memorial University of Newfoundland.

Zimmerer, K. S. (2018). Geography and the study of human-environment relations. In D. Richardson, N. Castree, M. F. Goodchild, A. Kobayashi, W. Liu, \& R. A. Marston (Eds.), International encyclopedia of geography. John Wiley \& Sons, Ltd. https://doi. org/10.1002/9781118786352.wbieg1028. 